Helgoländer wiss. Meeresunters. 32, 200-212 (1979)

\title{
Kompensation extremer Umwelteinflüsse bei Balanus balanus als Anpassung an die sessile Lebensweise
}

\author{
H.-H. SOMMER \\ Zoologisches und Vergleichend-Anatomisches Institut der Universität Bonn; \\ Poppelsdorfer Schloß, D-5300 Bonn, Bundesrepublik Deutschland
}

\begin{abstract}
Compensation for extreme environmental conditions in Balanus balanus as adaptation to the sessile mode of life. In the sessile barnacle Balanus balanus $\mathrm{L}$. the relationship between stimulus duration and reaction intensity varies considerably. Investigations on the light reactions (increased cirral activity per unit time) and shadow reactions (decreased cirral activity per unit time) showed that increase of stimulus duration at a temporal range of 1-5 min and 30-360 min causes an increased response intensity, whereas, at a range of 5-30 $\mathrm{min}$, the response intensity decreases. Two adaptive systems are assumed to be responsible for this behaviour. The peculiarities of the light and shadow reactions are interpreted as a result of interactions of central and peripheral components, but the unusual relationship between stimulus duration and reaction intensity is probably based on a greater influence of central components. The biological significance of the light and shadow reactions is discussed in relation to the larval and adult life: By means of the central variation of stimulus threshold in the described protective shadow responses $B$. balanus seems to compensate for extreme environmental conditions. The light responses observed in the adults are considered as resulting from metamorphosis of larval swimming activity.
\end{abstract}

\section{EINLEITUNG}

Für die Orientierung freibeweglicher Tiere ist in marinen Biotopen die Wechselwirkung zwischen hydrostatischem Druck, Licht und Temperatur ausschlaggebend. Das gilt vor allem für das Pelagial des offenen Wassers und des Küstenbereichs. In der lichtlosen und weitgehend temperaturkonstanten Tiefsee ist dagegen die Präferenz für beștimmte Tiefenbereiche offensichtlich nur von der Druckstärke abhängig (vgl. MacDonald, 1975). Für die vertikale Gliederung mariner Lebensräume hat der hydrostatische Druck wahrscheinlich die gleiche Bedeutung wie die Temperatur für die horizontale Gliederung im Oberflächenbereich der Ozeane in Pol-Äquator-Richtung (Schlieper, 1963).

In der vorliegenden Arbeit werden einige physiologische Wirkungen des Faktors Licht bei dem Cirripedier Balanus balanus L. beschrieben. Dabei wird versucht, durch die Verwendung dieser sessilen Art zwei grundsätzliche Probleme zu lösen: (1) Die Analyse fotokinetischer Reaktionen wird immer durch das gleichzeitige Auftreten fototaktischer Reaktionen erschwert (Fraenkel \& Gunn, 1961). Diese Schwierigkeit 
wird umgangen, wenn Versuchstiere ohne fototaktische Reaktionen, also sessile Tiere, benutzt werden. (2) Freibewegliche Tiere können störende Umwelteinflüsse mit lokomotorischer Aktivität kompensieren: Sie suchen optimale Regionen auf. Sessile Tiere müssen zur Kompensation störender Einflüsse andere Verhaltensweisen entwickeln, weil sie ihren Aufenthaltsort nicht wechseln können. Ein Beispiel dafür wird hier mitgeteilt.

Die Versuchstiere wurden verschiedenen Licht-Dunkel-Wechseln mit jeweils gleicher Länge der alternierenden LD-Zeiten ausgesetzt (LD 1:1 $\mathrm{min}$ bis LD 360:360 min). Ihr Verhalten unter der Einwirkung verschiedener Druck-Reizmuster soll in einer folgenden Arbeit beschrieben werden.

\section{MATERIAL UND METHODE}

Die untersuchten Exemplare von Balanus balanus L. stammten aus dem Fanggebiet "Tiefe Rinne" bei der Insel Helgoland. Die Experimente wurden im Wechsel zwischen vollständiger Dunkelheit und 1000 lx Belichtungsstärke durchgeführt. Die Versuchstiere lebten unter konstanten Bedingungen in Nordseewasser bei ständiger Strömung $\left(3,5 \mathrm{~cm} \cdot \mathrm{s}^{-1}\right.$; Wassertemperatur $15^{\circ} \mathrm{C}$; Salzgehalt: $34 \%$; Sauerstoffgehalt: $7,8 \%$; pH: 7,9; Wassertiefe: $25 \mathrm{~cm}$; Rauminhalt eines Versuchsaquariums: 2,5 1; umlaufende Wassermenge in den fünf parallel geschalteten Aquarien und der Filteranlage: 37,51$)$.

Das Prinzip der zur Aktivitätsregistrierung bei Seepocken entwickelten Methode ist an anderer Stelle bereits veröffentlicht worden (Sommer, 1967, 1972 a). Das sessile Versuchstier ist dabei mit senkrechter Bodenplatte (zusammen mit dem ausgesägten Stück des Substrates, das es mit seinem eigenen Gehäuse bedeckt) in einer Aktivitätsschaukel befestigt und schlägt mit seinen Zirren von unten nach oben (vgl. Sommer, 1977). Durch die bei jedem Zirrenschlag auftretenden Bewegungsimpulse wird die Schaukel jeweils ausgelenkt $\left(<1^{\circ}\right)$, und ein an ihr befestigter Zeiger unterbricht mit seinem Ende den Strahlengang einer Lichtschranke. Die dadurch ausgelösten Impulse werden verstärkt, registriert und gezählt (vgl. Abb. 6).

Als Maß für die Reaktionsstärke wurde die Zahl der Zirrenschläge pro Zeiteinheit gewählt. Zur grafischen Darstellung der Reaktionsstärke dient der Quotient aus der Differenz zweier Meßwerte zu ihrer Summe: $(a-b) /(a+b)$. a ist die Aktivitätsmenge innerhalb einer beliebig langen Zeit nach einem Reiz, $b$ ist die Aktivitätsmenge vor dem Reiz in einer gleich langen Zeit. Für $a=0$ ist $(a-b) /(a+b)=-1$; für $\mathrm{b}=0$ ist $(\mathrm{a}-\mathrm{b}) /(\mathrm{a}+\mathrm{b})=+1$. Damit können alle Meßwerte im Bereich zwischen +1 und -1 dargestellt werden (vgl. Sommer, 1978 a).

\section{ERGEBNISSE}

Die bei dem sessilen Krebs Balanus balanus L. nachgewiesene Belichtungsreaktion ist als Steigerung der motorischen Aktivität nach Steigerung der Belichtungsstärke meßbar, die Beschattungsreaktion als Hemmung der motorischen Aktivität nach Senkung der Belichtungsstärke. Die Belichtungsreaktion tritt in zwei Formen auf (Som- 
mer, 1977): (1) Wenn ein aktives Tier gereizt wird, steigert es die Frequenz des Zirrenschlages (Belichtungsreaktion I); (2) Wenn ein inaktives Tier gereizt wird, öffnet es kurze Zeit nach dem Reiz sein Gehäuse und beginnt mit dem Zirrenschlag (Belichtungsreaktion II). Nach v. Buddenbrock (1931) sollte die Stärke der Beschattungsreaktion ("Schattenreflex") nur von der Reizstärke, nicht aber von der Reizdauer abhängig sein. Die hier mitgeteilten Ergebnisse bestätigen die Abhängigkeit sowohl der Beschattungsreaktion als auch der v. Buddenbrock unbekannten Belichtungsreaktion von der Reizdauer.

\section{Abhängigkeit der Reaktionsstärke von der Reizdauer}

Wird die Reizdauer von $30 \mathrm{~min}$ ausgehend verlängert, nimmt auch die Reaktionsstärke zu (Sommer, 1978 b), wird die Reizdauer von $30 \mathrm{~min}$ ausgehend ver-
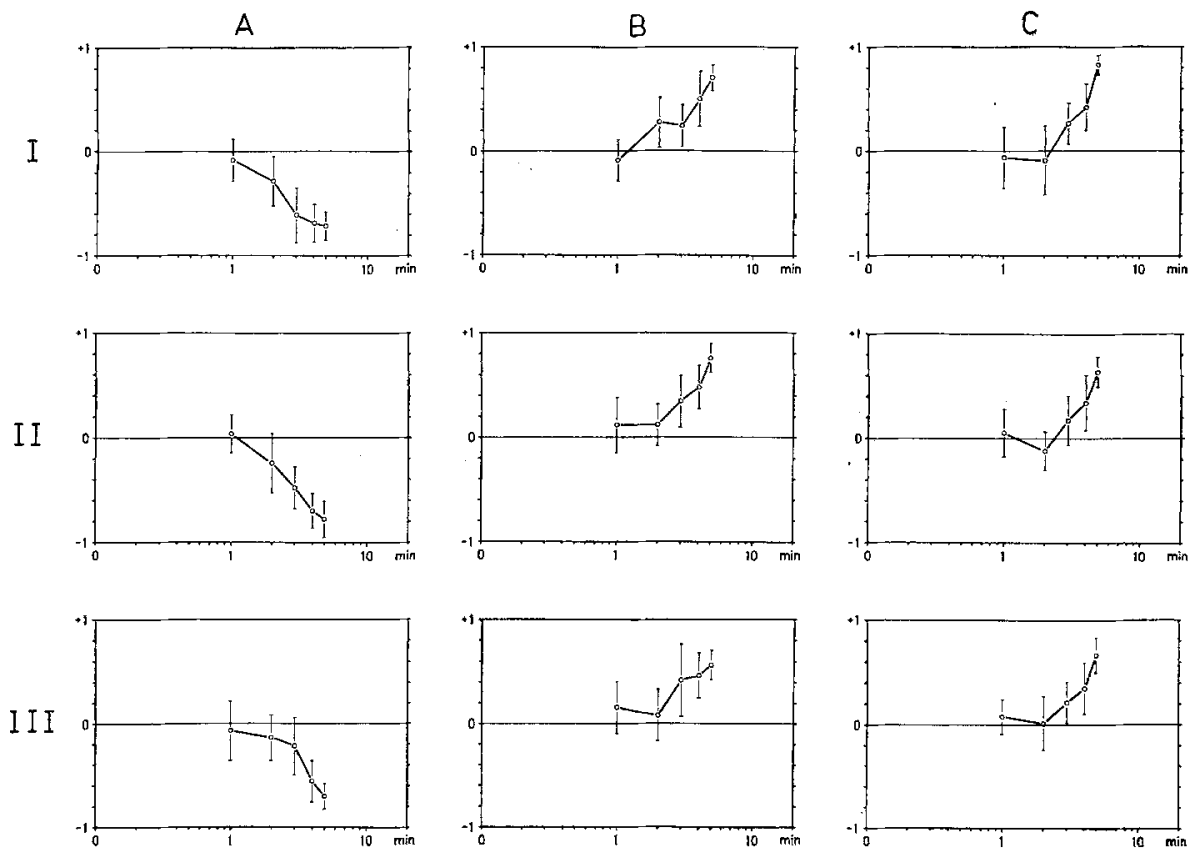

Abb. 1: Die Abhängigkeit fotokinetischer Reaktionen sessiler Krebse (Balanus balanus) von der Reizdauer bei raschem Wechsel der LD-Zeiten. Abszissen: Reizdauer (= Adaptationsdauer; min in logarithmischem Maßstab); Ordinaten: Reaktionsstärke (große oder kleine Aktivitätsmengen in den Hell- oder Dunkelzeiten, geprüft nach $5 \mathrm{~min}, 4 \mathrm{~min}, 3 \mathrm{~min}, 2 \mathrm{~min}$ und 1 min und berechnet mit dem Quotienten $(a-b) /(a+b)$. Liegen die Meßwerte im Bereich über der Nullinie, ist die Aktivitätsmenge in der Zeiteinheit nach dem Reiz größer als in einer gleich langen Zeit vor dem Reiz; liegen sie im Bereich unter der Nullinie, ist es umgekehrt. A: Beschattungsreaktion (Aktivitätspause); B: Belichtungsreaktion I (aktive Tiere werden gereizt; Steigerung der Schlagfrequenz); C: Belichtungsreaktion II (die Tiere werden $1 \mathrm{~min}$ nach Beginn einer spontanen Aktivitätspause vom Reiz getroffen). I, II, III: Drei Versuchstiere. Die Abweichung der Einzelwerte von den dargestellten Mittelwertkurven ist als Standardabweichung $s_{\overline{\mathrm{x}}}$ bei jedem Kurvenpunkt eingetragen. Versuchsdauter: $17 \mathbf{d}$ mit LD-Zeiten von jeweils $5 \mathrm{~min}(5 \mathrm{~d}), 4 \mathrm{~min}(4 \mathrm{~d}), 3 \mathrm{~min}(3 \mathrm{~d}), 2 \mathrm{~min}(3 \mathrm{~d})$ und $1 \mathrm{~min}(2 \mathrm{~d})$ Dauer 
kürzt, nimmt die Reaktionsstärke entgegen aller Erwartung ebenfalls zu (Sommer, 1978 a). Bei einer kürzeren Reizdauer als 5 min nimmt die Reaktionsstärke allerdings wieder ab (Abb. 1).

Die Abhängigkeit der Reaktionsstärke von der Reizdauer ist als schematische Ubersicht in Abbildung 2 dargestellt. Sie zeigt die $\mathrm{Zu}$ - und Abnahme der Reaktionsstärke mit zunehmender Reizdauer, doch ist festzuhalten, daß im Experiment die Reizdauer nicht von $1 \mathrm{~min}$ aus zunahm (LD 1:1 min bis LD 360:360 min), sondern von 30 min ausgehend nach rechts verlängert, nach links verkürzt wurde.

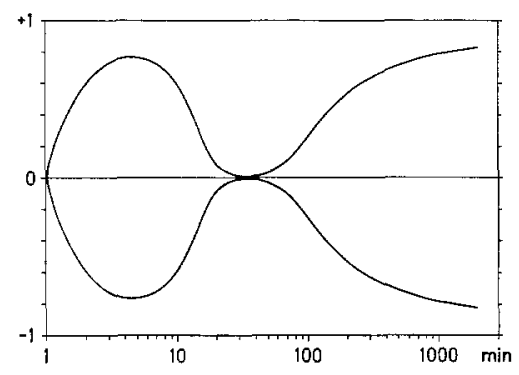

Abb. 2: Die Belichtungs- und Beschattungsreaktionen bei Balanus balanus. Die Abhängigkeit der Reaktionsstärke von der Reizdauer (schematische Darstellung). Abszisse: Reizdauer (= Adaptionsdauer; min in logarithmischem Maßstab); Ordinate: Reaktionsstärke (geprüft nach 1-5 min (Abb. 1), 5-30 min (Sommer, 1978 a), 30-360 min (Sommer, 1978 b). Auf der Ordinate ist im Bereich von 0 bis +1 die Stärke der Belichtungsreaktion, im Bereich von 0 bis -1 die Stärke der Beschattungsreaktion dargestellt

Zur Bestimmung der Reaktionsstärke mit dem Quotienten $(a-b) /(a+b)$ wurden in raschen LD-Wechseln (LD 1:1 min bis LD 10:10 min) die Aktivitätsmengen a und $\mathrm{b}$ in den jeweils gesamten $\mathrm{L}$ - und D-Zeiten herangezogen, in langsameren LD-Wechseln (LD 10:10 min bis LD 360:360 min) die Aktivitätsmengen a und b in jeweils $10 \mathrm{~min}$ vor und nach dem Wechsel der Belichtungsbedingungen. Die schematische Darstellung in Abbildung 2 ermöglicht folgende Formulierung des Ergebnisses: Mit zunehmender Reizdauer nimmt im Bereich von $1 \mathrm{~min}$ bis $5 \mathrm{~min}$ die Reaktionsstärke $\mathrm{zu}$, im Bereich von $5 \mathrm{~min}$ bis $30 \mathrm{~min} \mathrm{ab}$, im Bereich von $30 \mathrm{~min}$ bis $360 \mathrm{~min}$ wieder zu.

\section{Abhängigkeit der Aktivitätsverteilung von der Länge der L D - Z eiten}

Der ungewöhnliche Adaptionsverlauf bei Balanus balanus L. (Abb. 2) führt zu der Frage, wie die beschriebenen Reaktionsweisen in die Aktivität außerhalb des schützenden Gehäuses einbezogen sind. Die Verteilung der Aktivitätsäußerungen auf die Dunkel- und Hellzeiten während verschiedener LD-Wechsel ist in den Abbildungen 3, 4 und 5 dargestellt.

Die Abb. 3 zeigt, daß in langsamen LD-Wechseln (LD 12:12 h bis LD 1:1 h) Dunkelaktivität bevorzugt wird, jedoch bei einer Beschleunigung des LD-Wechsels 
über LD 30:30 min hinaus Übergang zur Hellaktivität erfolgt (die auf den Abszissen erfaßte Zeit ist nur ein kurzer Abschnitt länger dauernder Versuche). In Abbildung 4 ist ein Beispiel für Hellaktivität eines Tieres im LD-Wechsel von LD 10:10 min zu sehen; Abbildung 5 zeigt die Aktivitätsverteilung in LD-Wechseln von LD 4:4 min bis LD $1: 1 \mathrm{~min}$.
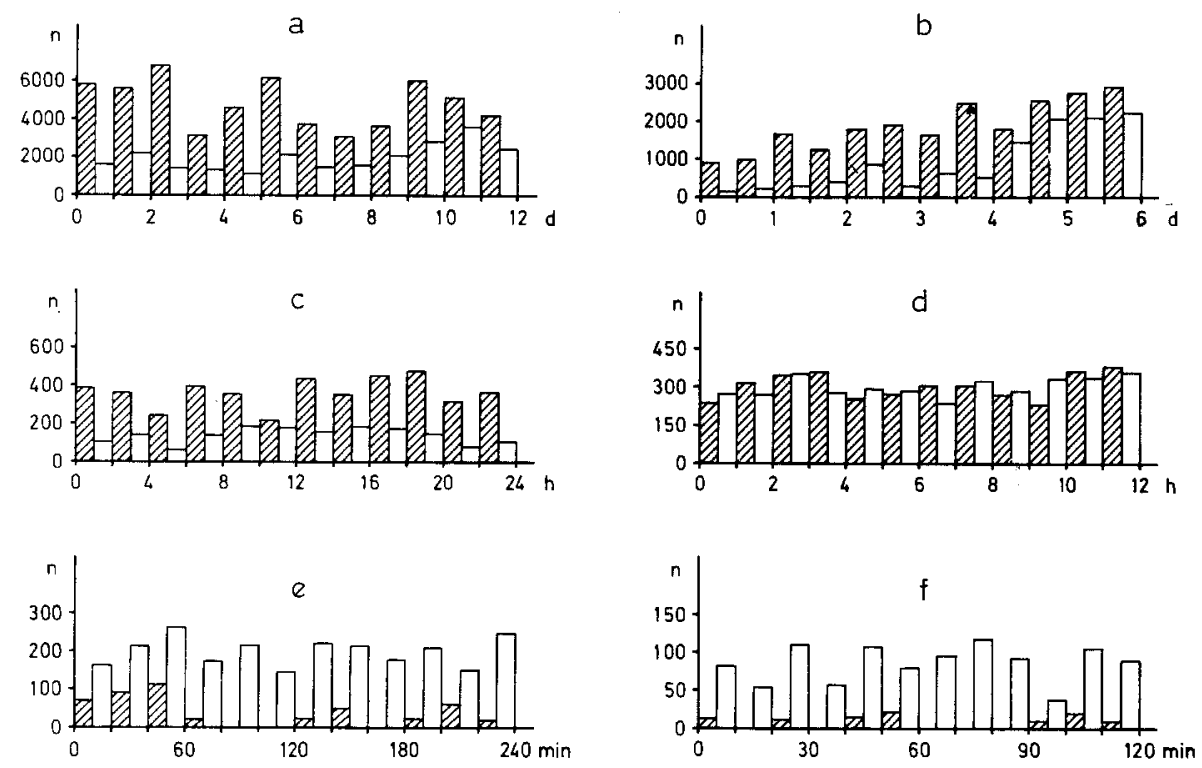

Abb. 3: Die Abhängigkeit der Aktivitätsverreilung eines Versuchstieres (Balanus balanus) von der Länge alternierender LD-Zeiten. Abszissen: Zeit in Tagen (d; $a, b)$, Stunden $(h ; c, d)$, Minuten (min; e, f) bei einer Länge der LD-Zeiten ron LD 12:12 h (a), 6:6 h (b), 1:1 h (c), 30:30 min (d), 10:10 min (e) und 5:5 min (f); Ordinaten: Aktivitätsmenge (n; Zahl der Zirrenschläge) in der Zeiteinheit. Die Aktivitätsmenge in der jeweils gesamten D- (schraffiert) oder L-Zeit (leer) ist als Säule eingetragen

Die Abbildungen 3 und 5 enthalten als direkte Umsetzung eines Protokolls von der Aktivität eines Einzeltieres die in Abbildung 2 zusammenfassend dargestellten Beziehungen zwischen Reaktionsstärke und Länge der LD-Zeiten (vgl. Abb. 7): In LD-Wechseln von LD 1:1 min und LD 30:30 min ergibt die Auswertung mit dem Quotienten $(a-b) /(a+b)$ Werte, die sich Null nähern (vgl. Abb. 1), d. h. in LD-Wechseln von ID 1:1 min gleichen sich die Unterschiede zwischen den Aktivitätsmengen a und $b$ in den LD-Zeiten aus, und in LD-Wechseln von LD 30:30 min stimmen die Aktivitätsmengen $a$ und $b$ sowohl $10 \mathrm{~min}$ vor und $10 \mathrm{~min}$ nach dem Umschalten als auch in den gesamten LD-Zeiten nahezu überein.

Beim Vergleich der Abbildung 6 mit den Aktivitätsmustern in Abbildung 5 e und $f$, die unmittelbar nach Abschluß der Versuche mit raschen LD-Wechseln registriert wurden, zeigt sich ein Nachlassen der Irritation, wenn die Tiere in Dauerlicht leben. Würde die in Abbildung 6 gezeigte Aktivität in den Koordinatensystemen der Abbildung 5 e und $f$ dargestellt, ergäbe sich eine gerade Linie in Höhe des Ordinatenwertes 35 (Zirrenschläge in der Minute). 


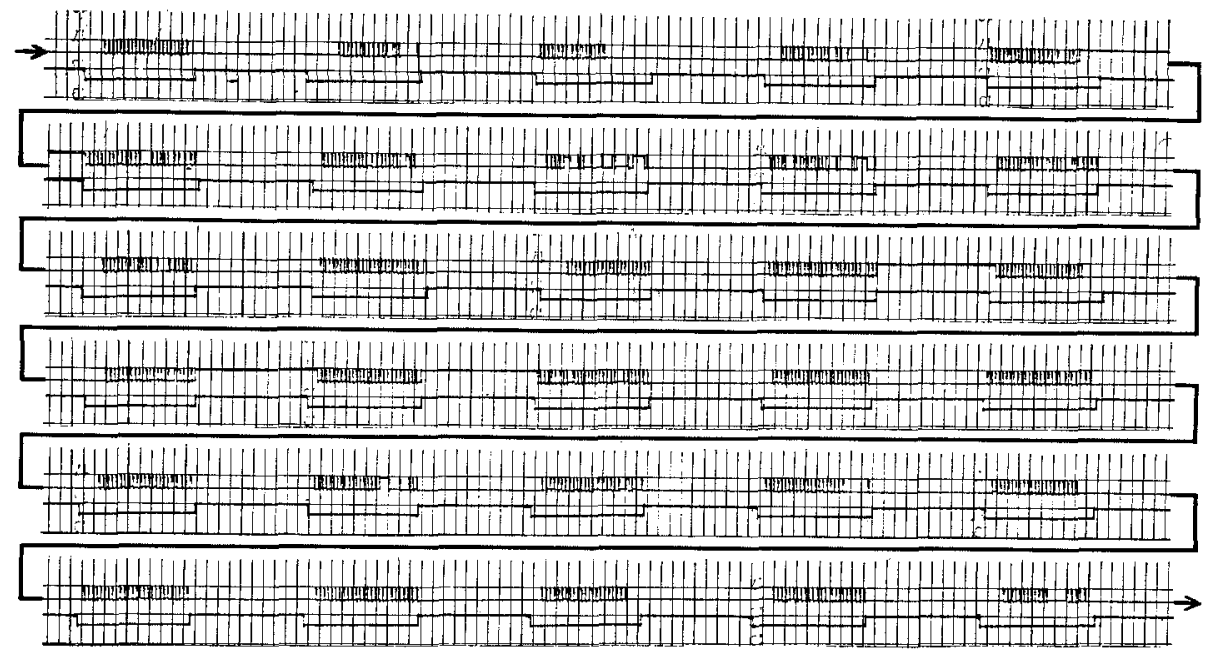

Abb. 4: Die motorische Aktivität einer Seepocke (Balanus balanus) im LD-Wechsel von ID 10:10 min (Foto eines Protokolls). Dargestellte Versuchsdauer: 10 h. Der Registrierstreifen ist in Minutenabständen geteilt, jeder Ausschlag des Ereignisschreibers entspricht 10 Zirrenschlägen des Versuchstieres. Die Umschaltpunkte der Belichtungsbedingungen sind unter der Registrierspur aufgezeichnet. Das Tier ist hellaktiv
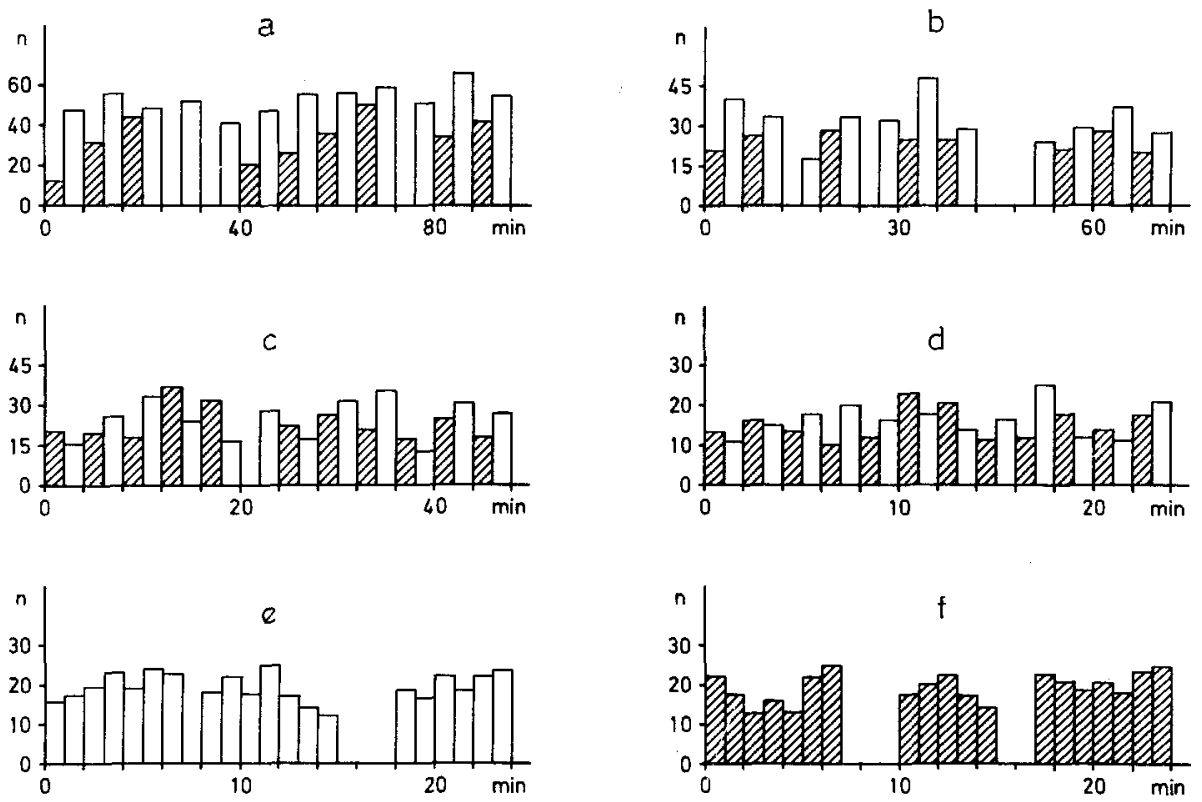

Abb. 5: Die Abhängigkeit der Aktivitätsverteilung eines Versuchstieres (Balanus balanus) von der Länge alternierender LD-Zeiten. Abszissen: Zeit in min bei einer Länge der LD-Zeiten von LD 4:4 min (a), 3:3 min (b), 2:2 min (c), 1:1 min (d) und in Dauerbedingungen LL (e), $\mathrm{DD}(\mathrm{f})$; Ordinaten: Aktivitätsmenge ( $\mathrm{n}$; Zahl der Zirrenschläge) in der Zeiteinheit. Als Säule ist die Aktivitätsmenge in der jeweils gesamten D- (schraffiert) oder L-Zeit (leer) eingetragen. Die Teilabbildung (e) zeigt das Aktivitätsmuster unmittelbar nach dem LD-Wechsel von LD 1:1 min, die Teilabbildung (f) das Aktivitätsmuster $60 \mathrm{~min}$ später. Die Unregelmäßigkeit des Aktivitätsmusters ist wahrscheinlich auf Irritation des Versuchstieres durch Überreizung zurückzuführen (vgl. Abb. 6) 


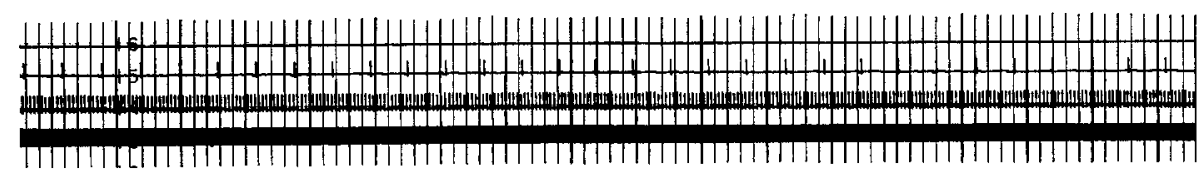

Abb. 6: Die Aktivität eines Versuchstieres (Balanus balanus) in Dauerlicht vier Tage nach Abschluß der Versuche mit raschen LD-Wechseln (max. LD 1:1 min). Foto eines Protokolls. Der Registrierstreifen ist in Minutenabständen geteilt. Jeder Ausschlag des Ereignisschreibers entspricht auf der oberen Spur 100, auf der mittleren Spur 10 und auf der unteren Spur einzelnen Zirrenschlägen des Versuchstieres. Die obere Leerspur dient zur Registrierung der Umschaltpunkte eines LD-Wechsels (vgl. Abb. 5 e u. f)

\section{Beziehungen zwischen den LD-Reaktionen und der LD-Aktivität}

Wie Abbildung 3 zeigt, sind Balaniden in LD-Cyclen von LD 12:12 $\mathrm{h}$ bis LD 1:1 h dunkelaktiv, d. h. die Individuen verlegen bis zu $80 \%$ ihrer Gesamtaktivität in die Dunkelzeiten. In den Dunkelzeiten nimmt also die Aktivitätsmenge in der Zeiteinheit zu, in den Hellzeiten ab.
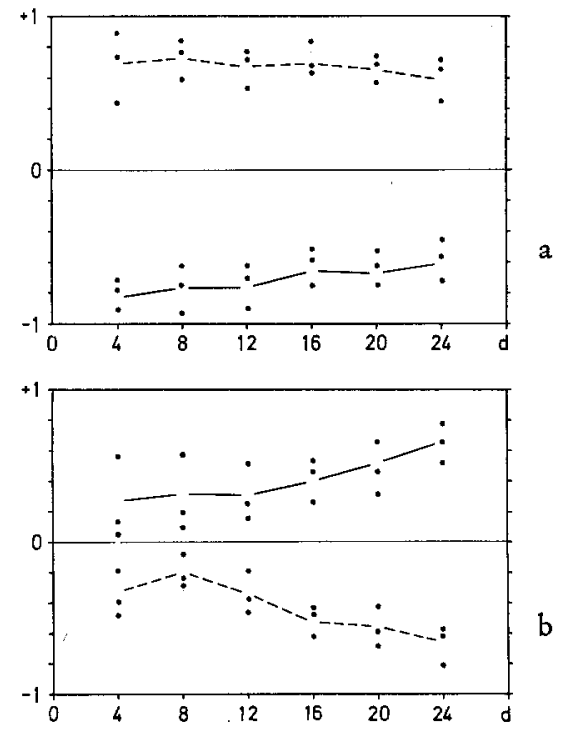

Abb. 7: Die Aktivitätsmenge eines Versuchstieres (Balanus balanus) $10 \mathrm{~min}$ (a) und $60 \mathrm{~min}$ (b) nach dem Wechsel der Belichtungsbedingungen in einem LD-Wechsel von LD 12:12 h. Abszissen: Versuchsdauer in Tagen (d) nach drei Wochen Aufenthalt in Dauerdunkel; Ordinaten: Aktivitätsmenge in der Zeiteinheit (geprüft nach $10 \mathrm{~min}$ (a) und $60 \mathrm{~min}$ (b), berechnet mit dem Quotienten $(a-b) /(a+b)$. Dargestellt ist die Veränderung der Aktivitätsmenge 10 min (a) und $60 \mathrm{~min}$ (b) nach dem Wechsel der Belichtungsbedingungen im Dunkeln (durchgezogene Linien) und im Licht (unterbrochene Linien; in a die Belichtungsreaktion II). Die Hemmung/ Stimulierung der Aktivität durch Beschattung/Belichtung (a) wird nach längerer Reizdauer (60 $\mathrm{min}$ in b) aufgehoben, so daß $60 \mathrm{~min}$ nach dem Wechsel der Belichtungsbedingungen Dunkelaktivität ausgeprägt ist (vgl. Abb. $3 \mathrm{c}$ ) 
Demnach verringert anhaltende Dunkelheit die durch plötzliche Intensitätsminderung der Belichtung ausgelöste Hemmung der Aktivität, anhaltende Belichtung die durch plötzliche Intensitätssteigerung ausgelöste Stimulierung der Aktivität. Eine Reizdauer von $1 \mathrm{~h}$ bei konstanter Reizstärke reicht aus, um diese Wirkung zu erzielen. Je länger ein LD-Wechsel dauert, um so deutlicher ist schon nach $1 \mathrm{~h}$ eindeutige LD-Aktivität ausgeprägt (ansteigende und abfallende Kurven in Abb. 7).

Nach den in Abbildung 7 dargestellten Ergebnissen haben konstante Belichtungsund Beschattungsreize die umgekehrte Wirkung plötzlicher Änderungen der Reizstärke. Plötzlich einsetzende LD-Reize stimulieren/hemmen die Aktivität, anhaltende LD-Reize hemmen/stimulieren die Aktivität der Versuchstiere.

\section{DISKUSSION}

Für die Entscheidung, den sessilen Krebs Balanus balanus zu untersuchen, waren folgende Uberlegungen ausschlaggebend: Die Wechselwirkungen zwischen den durch Licht und durch Druck ausgelösten physiologischen Vorgängen sind bei freibeweglichen Tieren wichtig für die Einhaltung einer jeweils optimalen Tiefenlage im Biotop (Rice, 1964). Auch die sessilen Tiere sind der Einwirkung von Druck und Licht ausgesetzt. Für die Analyse fotokinetischer Reaktionen sind sie besonders gut geeignet, weil sie keine fototaktischen Reaktionen zeigen, und für die Analyse von DruckReizwirkungen sind sie besonders gut geeignet, weil sie keine lokomotorische Aktivität haben. Sie können unter der Einwirkung konstanten Drucks untersucht werden. Konstante Versuchsbedingungen (und damit die Adaptation mariner Tiere) in verschiedenen Wassertiefen (Druckstärken) bei übereinstimmend konstanten Versuchsbedingungen waren bisher nicht möglich (vgl. Kinne, 1972; Flügel, 1972; MacDonald, 1975). Die Forderung, alle Versuchsbedingungen in verschiedenen Wassertiefen konstant $\mathrm{zu}$ halten, schließt die Verwendung von freibeweglichen Versuchstieren aus. Sie können durch lokomotorische Aktivität ihren Aufenthaltsort und damit auch die Druckbedingungen wechseln, denn der hydrostatische Druck im Schwerefeld hängt von der Höhe der Wassersäule über dem Versuchstier ab. Die Aktivität der Versuchstiere, der regelmäßige Zirrenschlag (vgl. Crisp \& Southward, 1961), ermöglicht die quantitative Erfassung der Reizwirkungen. Die sessile Lebensweise läßt vermuten, daß die Tiere besondere Anpassungen entwickelt haben, um extreme Umwelteinflüsse zu kompensieren (vgl. Einleitung); diese Vermutung wird durch die Ergebnisse der Analyse fotokinetischer Reaktionen bei Balanus balanus bestätigt.

Das in Abbildung 7 dargestellte Ergebnis besagt, daß konstante LD-Reize die umgekehrte Wirkung plötzlicher Änderungen der Reizstärke haben. Plötzlich einsetzende LD-Reize stimulieren/hemmen die Aktivität der Versuchstiere, konstante LD-Reize hemmen/stimulieren ihre Aktivität. Die Einwirkung konstanter LD-Reize während langsamer LD-Wechsel führt zu ausgeprägter Dunkelaktivität. Wahrscheinlich äußert sich darin auch bei Balanus balanus die Abneigung vieler Wasser- und Bodentiere gegen helles Licht (Aschoff, 1964; Müller, 1964). Dieser Aspekt ist für Balanus balanus in einer früheren Arbeit (Sommer, 1971) diskutiert worden.

Die Stärke der Belichtungs- und Beschattungsreaktionen ist nach plötzlichen Än- 
derungen der Reizstärke bei Balanus balanus nicht nur von der Reizstärke, sondern auch von der Reizdauer abhängig. Die Reaktionsstärke ändert sich jedoch mit zunehmender Reizdauer in unvorhergesehener Weise: Im Bereich von 1 min bis 5 min Reizdauer nimmt die Reaktionsstärke zu (Abb. 1), im Bereich von $5 \mathrm{~min}$ bis $30 \mathrm{~min}$ nimmt sie ab (Abb. 2; Sommer, 1978 a), und bei einer Verlängerung der Reizdauer über 30 min hinaus nimmt sie wieder zu (Abb. 2; Sommer, 1978 b). Im Bereich von $5 \mathrm{~min}$ bis $30 \mathrm{~min}$ Reizdauer widerspricht der beobachtete Zusammenhang von Reizdauer und Reaktionsstärke bisher bekannten Ergebnissen, nach denen zunehmende Reizdauer zunehmende Adaptation ermöglicht und damit bei einem Wechsel der Reizstärke auch zunehmende Reaktionsstärke bedingt.

Das auf den ersten Blick widersprüchliche Verhalten der Versuchstiere beruht vermutlich auf dem anteiligen Zusammenwirken von zwei Adaptationsvorgängen: Einem langsam ablaufenden (Langzeitadaptation) und einem schnell ablaufenden (Kurzzeitadaptation). Es scheint hier tatsächlich eine spezielle Anpassung vorzuliegen. Sie ermöglicht es den sessilen Tieren, auch auf extreme Umwelteinflüsse (in diesem Fall auf rasch wechselnde Reizstärken) sinnvoll zu reagieren: Die mit nachlassender Adaptation, auf Grund kürzerer Reizdauer ebenfalls nachlassende Reaktionsbereitschaft wird von den Versuchstieren wieder verstärkt. Der beobachtete Adaptationsverlauf spricht für einen stark endogen bedingten Anteil der gemessenen Reaktionen.

Ein Ansatzpunkt für die Interpretation der Ergebnisse ist die Frage: Welche physiologischen Vorgänge sichern den Schutz des Tieres durch ständige Reaktionsbereitschaft auch unter extremen Bedingungen? Das ist die Frage nach der biologischen Bedeutung der Beschattungsreaktion. Eigenarten der Belichtungsreaktion werden weiter unten diskutiert.

Das Verhalten der sessilen Versuchstiere kann zwischen zwei Polen eingeordnet werden: Aktivität außerhalb und Inaktivität innerhalb des schützenden Gehäuses. Die Aktivität außerhalb des Gehäuses dient einerseits zur Befriedigung der Stoffwechselbedürfnisse (Herbeistrudeln von Atemwasser und Zurückhalten fester Nahrung), macht aber andererseits das sessile Tier schutzlos.

Jeder potentielle Feind kann einen Schatten werfen, auf den das sessile Tier reagieren sollte. Wenn mit abnehmender Reizdauer, also zunehmender Belastung durch alternierende Reize, die Reaktionsstärke auf Grund unzureichender Adaptation immer mehr nachließe, wäre das sessile Tier bald nicht mehr geschützt. Nach den in Abbildung 2 zusammengefaßten Ergebnissen muß folgendes angenommen werden. Mit nachlassender Reizdauer wird die Schwelle zur Auslösung der Beschattungs-(Schutz-) reaktion immer höher. Der bei großer Reizbelastung lebenswichtige Schutz des Tieres wird dann in einem $Z$ wischenbereich ( $30 \mathrm{~min}$ bis $5 \mathrm{~min}$ Reizdauer) durch eine zentralnervös bedingte Schwellenerniedrigung sichergestellt. Der anschließend bei noch schnellerer Folge der LD-Reize und dadurch verkürzter Adaptationsdauer registricrte Schwellenanstieg entspricht dann wieder dem Adaptationsverlauf, wie er bei anderen Lichtsinnesorganen bekannt ist.

Einen vergleichbaren Vorgang beschrieb v. Buddenbrock (1931) bei Balanus (vgl. Sommer, 1977): Unmittelbar nach plötzlicher Belichtung steigt die Schwelle zur Auslösung des "Schattenreflexes" stark an und sinkt dann nach längerer Belichtungszeit auf einen stationären Wert ab. Dieser Verlauf ist dem Adaptationsverlauf bei ande- 
ren Lichtsinnesorganen entgegengesetzt. Dort steigt die Empfindlichkeitsschwelle bei der Helladaptation in der Regel langsam bis zum Endwert an, wie im menschlichen Auge, das bei der Adaptation Dunkel-Hell eine kontinuierliche Abnahme seiner Empfindlichkeit zeigt, bis es den Zustand der Helladaptation erreicht hat (Trendelenburg, 1961). Weil bei den Versuchstieren eine physikalische Minderung der Reizstärke nicht möglich ist (vgl. Kontraktion der Iris im Wirbeltierauge), findet bei ihnen unmittelbar nach der Belichtung eine zentrale Schwellenerhöhung statt.

Die Annahme von langsam und schnell ablaufenden Adaptationsvorgängen läßt es allerdings offen, welcher Vorgang peripher (in den Rezeptoren) und welcher zentral (neuronal) zu lokalisieren ist, doch wird die Bedeutung der neuronalen Basis durch Eigenarten der licht-elektrischen Energiewandlung in den Augen von Balanus gestützt (Krischer, 1973): Das mediane Auge reagiert deutlich schneller als die beiden lateralen Augen. Entstehung und Verarbeitung der Signale laufen bei Balanus räumlich voneinander getrennt $a b$, denn die Rezeptoren senden ihre elektrischen Signale über Nerven, die mehrere Millimeter lang sind, an das einfach gebaute Gehirn (im Wirbeltierauge erfolgt ein Teil der Signalverarbeitung bereits in der Netzhaut). Weil die Zellen des "schnellen" Auges wesentlich kleiner sind als die des "langsamen" Auges, hängt die Reaktionszeit offenbar auch von der Größe des Reaktionsvolumens ab. Nach Krischer (1972) ist die Geschwindigkeit der licht-elektrischen Energiewandlung, bezogen auf die Volumeneinheit der Rezeptorzellen, eine im Tierreich konstante Größe. Durch diese Überlegung wird die Annahme einer vorwiegend neuronal bedingten Grundlage der schnellen und langsamen Adaptationsvorgänge bei Balanus balanus wieder relativiert. Auch der diffuse Hautlichtsinn (Millot, 1957) ermöglicht vielen marinen Tieren wie Balanus (v. Buddenbrock, 1931) eindeutige Reaktionen auf Änderungen der Belichtungsstärke.

Es kann hier nicht entschieden werden, an welchen Orten langsame und schnelle Vorgänge ablaufen. Es kann nur gesagt werden: Wenn angenommen wird, daß je nach der Länge der Dunkel- und Hellzeiten ein Langzeit- oder ein Kurzzeitadaptationssystem mehr oder weniger beansprucht wird, dann kommt bei mittlerer Geschwindigkeit der LD-Wechsel (LD 30:30 min) offensichtlich ein Punkt, an dem beide Systeme gleich stark beansprucht werden, d. h. ihre Anteile lassen sich in den Reaktionen der Tiere nicht trennen.

Für den raschen LD-Wedhsel von LD 1:1 min müßte die gleiche Überlegung gelten, doch entspricht unter diesen Bedingungen das Verhalten der Tiere dem bekannten Zusammenhang von Reizdauer und Reaktionsstärke: Kurze Reiz(Adaptations-)dauer ermöglicht nur noch schwache Reaktionen, längere Reiz(Adaptions-)dauer ermöglicht stärkere Reaktionen. Unter dem Aspekt der biologischen Bedeutung ließe sich sagen, daß die Reaktionen auf Reize, die in rascher Folge und in gleicher Stärke wirken, für das Tier sinnlos wäre. Hier bewirkt demnach die ïbliche Beziehung zwischen Reizdauer und Reaktionsstärke, daß ein Tier davor geschützt wird, mechanisch auf jeden Reiz zu reagieren.

Immerhin ist der Gedanke an eine Trennung zentral und peripher bedingter Anteile am Zustandekommen der beobachteten Reaktionen berechtigt, denn vergleichbare Vorgänge, bei denen an der Bereichseinstellung lichtempfindlicher Rezeptoren zwei verschieden schnell adaptierende Systeme beteiligt sind, gibt es in den Netzhäu- 
ten der Wirbeltiere. Eingehende Untersuchungen zeigten (vgl. Trendelenburg, 1961), daß bei der Adaptation an schnell wechselnde Belichtungsbedingungen in Wirbeltieraugen ein schnell reagierendes System wirksam sein muß: Bei raschen LD-Wechseln kann der Belichtungsreiz als Störung der begonnenen Dunkeladaptation und der Verdunkelungsreiz als Störung der Helladaptation aufgefaßt werden. Beim Menschen verschlechtern Zwischenbelichtungen während der Dunkeladaptation (z. B. durch kurzes Einschalten der Beleuchtung im Versuchsraum) den Adaptationszustand nur wenig, wenn sie nur einige Sekunden dauern. Das ist nach Schober (1948) darauf zurückzuführen, daß die kurze Zwischenbelichtung nervöse Steuerungsvorgänge auslöst, die den Übergang von einem Adaptationszustand in einen anderen überbrücken, nämlich Änderungen der Pupillenweite und die von Schouten \& Ornstein (1939) untersuchte $\alpha$-Adaption, eine ungefähr $0,05 \mathrm{sec}$ dauernde, sofort einsetzende Empfindlichkeitsänderung der gesamten Netzhaut. Auf diese Weise wird durch die a-Adaptation eine Neueinstellung des Adaptationsniveaus, $d$. h. eine länger dauernde Anderung des Adaptationszustandes, vermieden. Die langsam ablaufende $\beta$-Adaptation beruht auf dem Konzentrationsverhältnis der Sehfarbstoffe zu ihren durch die Belichtung entstehenden Zerfallsprodukten (Schulze, 1968).

Die biologische Bedeutung der bei Balanus balanus festgestellten Belichtungsreaktion ist nicht so unmittelbar zu erkennen wie bei der Beschattungsreaktion, mit der sich das Tier durch Rückzug ins Gehäuse schützt. Die Aussage, das Tier verstärke seine Aktivität nach Belichtung, weil die Beschattung und damit eine Gefahr vorüber ist, befriedigt hierbei genau so wenig wie die Aussage, das Tier habe an der Beantwortung ständig wiederkehrender Reize ohne gefährliche Folgen "kein Interesse". Aussagekrältiger kann die folgende Interpretation sein: Die Belichtungsreaktion ist eine Metamorphose larvaler Schwimmaktivität. Diese Interpretation wird durch einige Uberlegungen gestützt: Viele marine Tiere verbringen einen Teil ihres Lebens in der euphotischen Zone. Wenn nicht passiver Auftrieb den Aufenthalt direkt unter der Oberfläche erzwingt (wie z. B. bei den Siphonophoren), erfolgt die Korrektur der jeweils optimalen Tiefenlage aktiv. Larven von Balanus reagieren auf Steigerung der Belichtungsstärke mit gesteigerter Schwimmaktivität negativ fotoaktisch (Bousfied, 1955). Negative Fototaxis wird bei Larven von Balanus balanoides auch durch Senken des hydrostatischen Drucks stimuliert (Reaktionstyp 3 b nach Rice, 1964). In den natürlichen Verhältnissen des Biotops entsprechen diese Verhaltensweisen der Kompensation passiven Aufsteigens an die Oberfläche. Abnehmende Belichtungsstärke und zunehmender, hydrostatischer Druck können das Gegenteil bewirken: Positive Fototaxis als Kompensation passiven Absinkens (vgl. Diskussion bei Sommer, 1977). Die freibeweglichen Larven reagieren demnach mit gesteigerter Schwimmaktivität auf einen Anstieg der Reizstärke und korrigieren dadurch ihre Tiefenlage. Die biologische Bedeutung dieses Verhaltens ist eindeutig. Das ist bei den sessilen Adulten nicht der Fall, obwohl sie auf einen Anstieg der Reizstärke ebenfalls mit gesteigerter Aktivität antworten. Deshalb bietet sich die Deutung an, die Belichtungsreaktion der sessilen Adulten sei eine Metamorphose larvaler Schwimmaktivität. Sollte die Interpretation zutreffen, dann wäre bei adulten Wirbellosen eine Verhaltensweise nachgewiesen, die den tiefgreifenden Wandel des gesamten Organismus während der Metamorphose überstanden hätte. Außerdem ist mit der Belichtungsreaktion eine Verhaltensweise 
beschrieben, die eine Alternative zu den bisher bekannten Schutzreaktionen (v. Budcenbrock, 1952; Sommer, 1977) ist. Belichtungs- und Beschattungsreaktionen zusammen sind zwei zueinander antagonistische Verhaltensweisen (stimulierte/gehemmte Aktivität), die dem Antagonismus der auslösenden Reize (Belichtung/Beschattung) entsprechen. Dieser Antagonismus ist bei bodenbewohnenden marinen Wirbellosen nicht selbstverständlich. Das häufigste Schutzverhalten ist Inaktivierung bis hin zur Bewegungsstarre. Die Tiere reagieren dabei auf Abnahme der Belichtungsstärke (Beschattung) oder/und auf Zunahme der Belichtungsstärke (Belichtung). Weil hier qualitativ verschiedene Reize (Beschattung und Belichtung) qualitativ gleichartige Reaktionen (Schalenschluß, Bewegungsstarre) auslösen, führt die konsequente Bezeichnung der Reaktionen nach der Herkunft der Reize zu Widersprüchen, denn in der Regel lösen im Tierreich qualitativ verschiedene Reize auch qualitativ verschiedene Reaktionen aus (z. B. Aktivierung und Inaktivierung bei tag- oder nachtaktiven Tieren). Auch bei bodenbewohnenden marinen Wirbellosen sind als Alternative zur Inaktivierung durch Beschattung und Belichtung Aktivierungsreaktionen auf Änderungen der Reizstärke denkbar. Dafür ist als ein Beispiel die Belichtungsreaktion von Balanus balınus beschrieben worden (Sommer, 1977). Der Antagonismus der Belichtungs- und Beschattungsreaktion ist die Grundlage der hier vorliegenden Ergebnisse.

Sinnvolle Aussagen über das Verhalten der Tiere im Biotop sind erst möglich, wenn ihr Verhalten auch unter der Einwirkung von Druck-Reizmustern bekannt ist. Die getrennte Untersuchung von Licht- und Druck-Reizwirkungen kann dabei nur der Anfang sein, denn bei der Kopplung von Ergebnissen, die dadurch gewonnen werden, ist gegenüber der Faktorengesamtheit im natürlichen Biotop eine Aussage uiber ihr Zusammenwirken schwierig. Darum muß versucht werden, die zunächst getrennt verwendeten Reize (vgl. Sommer, 1967-1978) auch schon unter Laborbedingungen zu koppeln. Dadurch könnte ein Beitrag zur Klärung physiologischer Grundlagen der vertikalen Gliederung mariner Lebensräume geleistet werden.*

\section{ZITIERTE LITERATUR}

Aschoff, J., 1964. Die Tagesperiodik licht- und dunkelaktiver Tiere. Revue suisse Zool. 71, 538-558.

Bousfield, E. L., 1955. Ecological control of the occurrence of barnacles in the Miramidi Estuary. Bull. natn. Mus. Can. 137, 1-69.

Buddenbrock, W. v., 1931. Untersuchungen über den Schattenreflex. Z. vergl. Physiol. 13, 164-213.

- 1952. Vergleichende Physiologie. Bd 1: Sinnesphysiologie. Birkhäuser, Basel, 504 pp.

Crisp, D. J. \& Southward, A. J., 1961. Different types of cirral activity of barnacles. Phil. Trans. R. Soc. (B) 243, 271-308.

Flügel, H., 1972. Pressure - Animals. In: Marine ecology. Ed. by O. Kinne. Wiley-Interscience, London, 1 (3), 1407-1450.

Fraenkel, G. S. \& Gunn, D. L., 1961. The orientation of animals. Dover Publ., New York, $376 \mathrm{pp}$.

Kinne, O., 1972. Pressure - General introduction. In: Marine ecology. Ed. by O. Kinne, Wiley-Interscience, London, 1 (3), 1323-1360.

* DFG-Projekt So $78 / 2$ 
Kries, J. v., 1894. Über den Einfluß der Adaptation auf Licht- und Farbempfindung und über die Funktion der Stäbchen. Ber. naturf. Ges. Freiburg i. Br. 9 (2).

Krischer, C. C., 1972. On the mechanism of electric response of the photoreceptors of the barnacle and other animals. Z. Naturf. 27 b, 409-413.

- 1973. Licht-elektrische Energiewandlung in den Augen der Seepocke. Umschau 73, 667-668.

MacDonald, A. G., 1975. Physiological aspects of deep sea biology. Cambr. Univ. Press, London, $450 \mathrm{pp}$.

Millot, N., 1957. Animal photosensitivity, with special reference to eyeless forms. Endeavour 16, 19-28.

Müller, H. J., 1964. Autökologie terrestrischer Wirbelloser: Der Faktor Licht. Fortschr. Zool. 16, 500-523.

Rice, A. L., 1964. Observation on the effects of changes of hydrostatic pressure on the behaviour of some marine animals. J. mar. biol. Ass. U. K. 44, 163-175.

Schlieper, C., 1963. Neuere Aspekte der biologischen Tiefseeforschung. Umschau 15, 457-461.

Schober, H., 1948. Die Nachtmyopie und ihre Ursachen. Albrecht v. Graefes Arch. Ophthal. $148,171$.

Schouten, J. F. \& Ornstein, L. S., 1939. Measurements on direct and indirect adaptation by means of a binocular method. J. opt. Soc. 29, 168.

Schulze, J., 1968. Elektroretinographische Untersuchungen an Fischaugen zum Problem der Readaptation und der Gültigkeit des Bunsen-Roscoeschen Gesetzes. Z. vergl. Physiol. 59, 250-271.

Sommer, H.-H., 1967. Aktivitätsperiodik bei Seepocken. Naturwissenschaften 54, 347-348.

- 1971. Exogene Steuerung der motorischen Aktivität von Balanus balanus L. durch Aktivierung zweier Adaptationssysteme des Lichtsinns. Verh. dt. zool. Ges. 65, 271-275.

- 1972 a. Endogene und exogene Periodik in der Aktivität eines niederen Krebses (Balanus balanus L.). Z. vergl. Physiol. 76, 177-192.

- 1972 b. Untersuchungen zur Physiologie der Druckwahrnehmung an sessilen Krebsen (Balanus balanus und Balanus improvisus) des Sublitorals und Eulitorals. Helgoländer wiss. Meeresunters. 23, 352-364.

- 1972 c. Untersuchungen zum Nachweis spezifischer Perzeptionsmechanismen im Drucksinn sessiler Krebse (Balanus balanus L. und Balanus improvisus Darwin). Experientia 28, 1149-1150.

- 1977. Die Belichtungsreaktionen sessiler Crustaceen (Balanus balanus) als Alternative zu den Schutzreaktionen bodenbewohnender mariner Wirbelloser. Helgoländer wiss. Meeresunters. 29, 493-502.

- 1978 a. Belichtungs- und Beschattungsreaktionen bei Balanus balanus L. (Cirripedia). Die Abhängigkeit der Reaktionsstärke von der Reizdauer in raschen LD-Wechseln. Z. Naturf. 33 e, $452-454$.

- 1978 b. Belichtungs- und Beschattungsreaktionen bei Balanus balanus. Die Abhängigkeit der Reaktionsstärke vom Adaptationszustand. Helgoländer wiss. Meeresunters. 31, 298-302.

Trendelenburg, W., 1961. Der Gesichtssinn, Springer, Berlin, 440 pp. 